\title{
Exploring blockchain technology and its potential applications for education
}

\author{
Guang Chen ${ }^{1,2}$, Bing Xu' , Manli Lu and Nian-Shing Chen ${ }^{3^{*}}$
}

\author{
* Correspondence: \\ nschen@mis.nsysu.edu.tw \\ ${ }^{3}$ Department of Information \\ Management, National Sun Yat-sen \\ University, Kaohsiung, Taiwan \\ Full list of author information is \\ available at the end of the article
}

\begin{abstract}
Blockchain is the core technology used to create the cryptocurrencies, like bitcoin. As part of the fourth industrial revolution since the invention of steam engine, electricity, and information technology, blockchain technology has been applied in many areas such as finance, judiciary, and commerce. The current paper focused on its potential educational applications and explored how blockchain technology can be used to solve some education problems. This article first introduced the features and advantages of blockchain technology following by exploring some of the current blockchain applications for education. Some innovative applications of using blockchain technology were proposed, and the benefits and challenges of using blockchain technology for education were also discussed.
\end{abstract}

Keywords: Blockchain, Educational evaluation, Instructional design, Learning is earning

\section{Background}

Blockchain is the core technology used to create the cryptocurrency, Bitcoin, through the maintenance of immutable distributed ledgers in thousands of nodes proposed by Satoshi Nakamoto in 2008 (Nakamoto 2008). It has been considered as part of the fourth industrial revolution since the invention of steam engine, electricity, and information technology (Chung and Kim 2016; Schwab 2017). This disruptive technology will have a significant impact on national governance, institutional functions, business operations, education, and our daily lives in the $21^{\text {st }}$ century. It has the potential to transform the current Internet from "The Internet of Information Sharing" to "The Internet of Value Exchange." Blockchain technology is expected to revolutionize the operating modes of commerce, industry, and education, as well as to promote the rapid development of knowledge-based economy on a global scale. Due to its immutability, transparency, and trustworthiness for all transactions executed in a blockchain network, this innovative technology has many potential applications (Underwood 2016). During the initial stages of its appearance, blockchain technology was not able to draw a lot of attention. However, as Bitcoin continues to run safely and steadily over the years, the society has since become aware of the enormous potential of the underlying technology of this invention in its application to not only cryptocurrency but also in many other areas (Collins 2016). Blockchain technology has become a hot topic for more and more countries, institutions, enterprises, and researchers. 
Presently, blockchain technology has been applied in various fields such as cryptocurrencies in the financial area, which includes Bitcoin, Ethereum, and Zcash (Zerocash), etc. Bitcoin is the first peer-to-peer payment network of electronic cash based on the blockchain technology. One of the crucial features of blockchain technology is how many nodes in a distributed blockchain network maintain consensus and the Bitcoin blockchain network adopts a hash-based Proof-of-Work (PoW) distributed consensus algorithm (Nakamoto 2008). Ethereum is an open-source, public, blockchain-based distributed computing platform featuring smart contract functionality using proof-of-stake consensus algorithm (Beck et al. 2016). Zcash is a decentralized and open-source cryptocurrency like Bitcoin. However, it offers a better privacy and selective transparency of transactions by using proof-of-zero-knowledge consensus algorithm. Zcash payments are published on a public blockchain, but the sender, recipient, and amount of a transaction remain private (Peck 2016). Besides, some organizations and enterprises are also trying to develop decentralized platforms based on blockchain technology. For example Arcade City, so-called "Uber Killer," is a ride-sharing company that has integrated its model in Ethereum, including identity and reputation systems (Zheng et al. 2017). Ubitquity is a digital property management company that offers secure recording and tracking records built on a blockchain platform.

Swan (2015) indicated that the development of blockchain applications could be divided into three stages; Blockchain 1.0, 2.0, and 3.0. Blockchain 1.0 is the deployment of cryptocurrencies as a peer-to-peer cash payment system. Blockchain 2.0 is the extensive blockchain applications than simple cash transactions, including stocks, bonds, loans, smart property, and smart contacts. Blockchain 3.0 is developing blockchain applications beyond currency, finance, and markets, such as in the areas of government, health, science, literacy, culture, and art.

According to the previously mentioned principle, the current applications of blockchain is still in the 1.0 and 2.0 stages. Most people do not know about the term "blockchain," not to mention the potential applications of using blockchain technology. Although researchers discussed the usage of blockchain in the commercial area (Swan 2015), several studies focused on how blockchain technology can be applied in education (Devine 2015; Sharples and Domingue 2016).

The remainder of this article will discuss the critical terminologies of blockchain technology such as "distributed ledger," "block and chain," and "the verification mechanism." In section 3, the current applications of blockchain technology in education is reviewed. Following on to the four innovative applications of using blockchain technology in education and the advantages are presented.

\section{Literature review}

What is blockchain?

Blockchain technology is also known as distributed ledger technology. It allows participants to secure the settlement of transactions, achieve the transaction, and transfer of assets at a low-cost (Tschorsch and Scheuermann 2016). A sample flow of cryptocurrency blockchain transaction can be seen as follows. User A initiates a transaction to User B via a peer-to-peer blockchain network. A cryptographic proof of identity (a pair of public key and private) is used to the network to identify user A and user B uniquely. 
The transaction will then be broadcasted to the memory pool of the blockchain network waiting for transaction verification \& validation. The new block is generated by obtaining a certain number of approved nodes; this is called reaching consensus. After reaching consensus, new "block" on the entire blockchain network is formed, and each node updates its respective copy of the blockchain ledger. This block contains all the transactions that occurred during this time. It is "linked" to the original block in the network through the digital signature (Yli-Huumo et al. 2016). The consensus stage is achieved through the use of a consensus algorithm. This process is called mining. Namely, Peer-to-Peer network reaches consensus on the current state of the distributed ledger (Kraft 2016). Each node can vote through its CPU power to accept valid blocks by taking extensions or reject invalid blocks by denying expansions. Any required rules and incentives can be implemented through this consensus mechanism (Nakamoto 2008). Each transaction in a block is tagged by a specific timestamp. The two blocks are also linked by a timestamp. Therefore, the data on the blockchain has a property of time, and the length of the chain is continuously growing. It means that blockchain is a distributed variant that implements the timestamp service (Haber and Stornetta 1991). Blockchain uses specialized hardware to construct sizeable cryptographic data chain, and SHA-256 hash function is used to prevent the tampering of data of third-party users (Tschorsch and Scheuermann 2016). Any attempt to change even just a bit of information will break the existing chains. In short, blockchain is a decentralized and trustworthy digital public ledger. It uses distributed techniques and consensus algorithms that were maintained by all participants.

Blockchain is not only a new type of internet infrastructure based on distributed applications but also a new type of supply chain network. Essentially, blockchain is a distributed network of computers (nodes) used to maintain the source of information sharing. Each node maintains the security and accuracy of the information by keeping a complete set of ledgers of past transactions. When a new block is being created by a miner, who is the first one to validate all the transactions in the block and solve the mathematical problem by generating a digital signature for the block which meets a pre-defined rule using the hash function. The newly created block will be broadcasting to the whole blockchain network, allowing all nodes to maintain the same complete ledger (Tschorsch and Scheuermann 2016).

Consensus mechanism is achieved through three major verification mechanisms. Bitcoin uses a verification mechanism called Proof of Work (Nakamoto 2008). The miners are nodes working in a blockchain peer-to-peer network. Their task is to validate all transactions included in one block and solve the mathematical problem of the digital signature using a hash function. The miners compete with each other, and once someone solves the problem, the solution will be shared with other mining nodes. The winning miner receives additional bitcoins as rewards. Other miners accept the Proof of Work, and the new block will be added to the blockchain network (Fanning and Centers 2016). Ethereum has four development stages, including Frontier, Homestead, Metropolis, and Serenity. The first three stages use the verification mechanism of Proof of Work, and the fourth stage uses Proof of Stake. The Proof of Stake requires the certifier to show the ownership of a certain amount of cryptocurrency (Sharples and Domingue 2016). "Proof of Zero Knowledge" is the consensus mechanism used in Zcash which can provide better privacy to its users. 
Compared with other verification mechanisms, Proof of Zero Knowledge has improved both regarding functionality and efficiency (Tschorsch and Scheuermann 2016).

\section{Features of blockchain technology}

From the technical point of view, blockchain technology has four features: decentralization, traceability, immutability, and currency properties.

Decentralization refers to the processes of data verification, storage, maintenance, and transmission on blockchain which are based on a distributed system structure. In this structure, the trust between distributed nodes is built through mathematical methods rather than the centralized organizations.

Traceability means that all transactions on blockchain are arranged in chronological order, and a block is connected with two adjacent blocks by the cryptographic hash function. Therefore, every transaction is trackable by examining the block information linked by hash keys.

There are two reasons that blockchain technology is immutable. On the one hand, all transactions are stored in blocks with one hash key linking from the previous block and one hash key pointing to the next block. Tampering with any transaction would result in different hash values and would thus be detected by all the other nodes running precisely the same validation algorithm. On the other hand, blockchain is a shareable public ledger stored on thousands of node, and all ledgers continue to sync in real time. Successful tampering would need to change over $51 \%$ of the ledgers stored in the network (Tschorsch and Scheuermann 2016).

Blockchain technology and cryptocurrency are inseparable, that is to say, any blockchain network has a form cryptocurrency property. The essence of blockchain technology is point-to-point transactions, no third party is involved, which means that all transactions do not require the participation of third parties. Circulation of digital currency based on blockchain technology is fixed. Specifically, in Bitcoin, the currency base is set at 21 million caps, so the generation of digital currency is created by using a specific mining algorithm and is bounded by a pre-defined formula. Thus there won't be the problem of inflation, collapse and so on. In Blockchain 2.0 and 3.0 applications, the combination of other activities such as government activities, educational activities, and financial activities can make these non-financial activities have the property of currency.

\section{Advantages of blockchain technology}

Derived from the above mentioned four technical features, some advantages of their application using blockchain technology are described as follows.

Reliability: the decentralized nature of a blockchain network changes the databases of the entire transaction records from closed and centralized ledgers maintained by only a few accredited institutions to open distributed ledgers maintained by tens of thousands of nodes. The failure of a single node does not affect the operation of the whole network. This avoids the single point of failure and ensures the high reliability of the applications which built on the blockchain technology.

Trust: blockchain network makes the trust decentralized too. Unlike the centralized trust we take for granted, such as central governments issuing currencies and 
commercial banks, blockchain network acts as new trust bearers with decentralized ledgers. These ledgers are shared among a network of tamper-proofed nodes (Underwood 2016).

Security: blockchain network uses the one-way hash function which is a mathematical function that takes a variable-length input string and converts it into a fixed-length binary sequence. The output bears no apparent relationship to the input. The process is hard to reverse because, given just the output, the input is impossible to determine (Yli-Huumo et al. 2016). Furthermore, the newly generated block is strictly following the linear sequence of time.

Efficiency: all data are automatically run through pre-set procedures. Therefore, blockchain technology can not only significantly reduce the cost of labor but also improve efficiency. For the digital currency of Blockchain 1.0, the automation of distributed ledger is mainly the automation of settlement. Blockchain technology could speed the clearing and settlement of certain financial transactions by reducing the number of intermediaries involved, and by making the reconciliation process faster and more efficient (Wang et al. 2016).

\section{Educational applications using blockchain technology \\ Present blockchain applications in education}

Nowadays, some universities and institutes have applied blockchain technology into education, and most of them use it to support academic degree management and summative evaluation for learning outcomes (Sharples and Domingue 2016; Skiba 2017).

Blockchain technology can formulate the whole transcript. In the formal learning context, this includes learning contents and outcomes as well as students' achievements and academic certificates. Subsequently, in the informal learning context, information about research experience, skills, online learning experience as well as individual interests are included. These data can be safely stored and accessed on a blockchain network in appropriate ways. The University of Nicosia is the first school which uses blockchain technology to manage students' certificates received from MOOC platforms (Sharples and Domingue 2016). Sony Global Education also used the blockchain technology to create a global assessment platform to provide services for storing and managing degree information (Hoy 2017). Additionally, Massachusetts Institute of Technology (MIT) and the Learning Machine company cooperated to design a digital badge for online learning based on blockchain technology. Students who have attended the projects of MIT Media Lab and passed the assessment will receive a certification which will be stored on a blockchain network (Skiba 2017). What's more, Holberton School is the first institute applying blockchain technology to store degrees and has claimed that they would share this information from 2017. The blockchain ledger can match all kinds of educational information with the user's unique ID. It includes learning behavior in class, micro academic project experience, and macro educational background, etc.

Moreover, blockchain technology contributes to reducing degree fraud. In the past, there were numerous cases of degree fraud. However, it can be avoided by employing blockchain in granting and managing student's degree now. The data matched with users' ID and stored in blockchain are checked, validated, and maintained by the 
miners from all over the world. Blockchain distributed ledger is immutable and trustworthy. Thus, the reliability and authority are both ensured, which will significantly reduce degree fraud.

Also, blockchain can be used as a "capacity-currency transformation bank." Specifically, blockchain learning ledger records detailed information about the users' learning experience and follows the development of their knowledge and skills. All of them can be transformed into a sort of digital currency and stored on a blockchain network according to a series of comprehensive standards. Students will gain rewards through their efforts on studies, which is called "learning is earning" (Sharples and Domingue 2016). Some schools have also started the application with this concept, for example, Sharples and Domingue (2016) claimed a kind of Education Reputation Currency named Kudos. It can be used to measure learning outcomes and stored in a virtual wallet.

\section{Future innovative educational applications using blockchain technology}

Blockchain technology can be applied to education in many innovative ways beyond just diploma management and achievements assessment. For both learners and teachers, blockchain technology has a great potential for broader application prospects on formative evaluation, learning activities design and implementation, and keep tracking the whole learning processes. Some innovative applications of using blockchain technology in the field of education are proposed as follows.

A Smart Contract running on the Ethernum blockchain network is essentially a computer protocol that simulates a real contract (such as economic transactions, employment, etc.) (Kosba et al. 2016). It can facilitate contract negotiation, simplify contract terms, implement contract execution, and verify contract fulfillment state. It marks the unique and precise identity of parties in a transaction (contract subjects) through a digital way and stipulates the rights and obligations of both sides (contract terms) by code. The smart contract not only reduces "third party costs" in traditional transactions but also dramatically guarantees the transaction security and reliability. For example, in the context of car-installment, the buyer negotiates with the seller directly rather than loans from the bank, saving any additional processing fees. If buyer breaks the rules, the code will be executed, and the smart contract will be terminated. The smart contract greatly improves executive power and fairness than the traditional one. Therefore, if teachers and students carry out instructing and learning activities based on a smart contract, some of the educational issues would be solved.

From the perspective of students, there are still some negative subjective or objective factors causing poor learning outcomes, such as the lack of motivation and financial pressure. Due to the trait of currency property, blockchain can be used to motivate students by implementing "learning is earning" (Sharples and Domingue 2016). The smart contract between teachers and students can be applied to the educational scenario. Real-time awards can be given to students through some simple clicks by the instructors. Students will get a certain number of digital currency according to smart contract as rewards. This kind of money can be stored in the education wallet, used as tuition, even exchanged with real currencies. 
Evaluation is also a problematic issue in the education system. Formative assessment has been advocated for a long time, and yet it is still not ripe because it is not easy to track every detail of teaching and learning. Applying blockchain and smart contract can cope up with this challenge. Notably, the immutability, traceability, and reliability of blockchain mean that the data recorded on blockchain are more specific, authentic, and anti-theft. Take the "collaborative learning" for instance, which is regarded as an excellent way to carry out constructivism instruction and cultivate students' ability to work with others. However, it is often accompanied by the problem of free-riding hindering fair evaluation. Blockchain technology can mitigate this phenomenon. Each student submits his/her work to the learning platform through his/her unique account, the smart contract running on it will review student's performance, and the results will be recorded into blocks. All behaviors during collaboration will be saved into blocks as evidence for evaluation as well. Moreover, public blockchain has the trait of decentralization. It means that the distributed ledger ensures the consistency of most nodes. Thus, as nodes in blockchain network, students' opinions would be taken into consideration when assessing them. In this context, blockchain ensures the fairness of the evaluation.

From the perspective of teachers, the instruction is sophisticated and artistic so that it is difficult to evaluate. The traditional method based on students' feedback tends to be one-sidedness, lacking subjectivity and is hardly helpful for teachers' improvement. A new assessment system can be constructed based on blockchain network and smart contract. First, teachers need to submit preplanned instructional activities as a smart contract to the schools. During the teaching process, all teaching activities will be recorded in the blockchain network. The smart contract will verify the consistency of the teaching design and practice, which is going to be an important instruction evaluation indicator. What's more, a smart contract between teachers and schools, as well as the one between teachers and students can be verified and supplemented with each other. Teachers who meet the standards will get digital currency as a reward. It serves as both an appreciation and encouragement for teachers' teaching skills.

From the perspective of student development, supervisor or academic advisor is directly responsible for the supervision of the student's program. They have the responsibilities of assisting the student in planning study programs and staying informed of student's research activities and progress. However, in practice, these issues are not checked and supervised, so it will be controversial to distinguish the responsibilities if something negative happens in the future. This situation will be changed if smart contract and blockchain technology is used in this area. All details should be monitored by smart contract platform and recorded into blockchain ledger. Such as how many times has the supervisor discussed with students in the past semester? How many times has the supervisor reviewed the thesis both in draft and final form? Whether they provide appropriate guidance to the students in course selection and research design? Thanks to the traceability and immutability of blockchain technology, both students and supervisors' behaviors will be recorded in the blockchain ledger. This innovative application can protect the interests of both parties.

Overall, blockchain can be used to construct a balance to measure learning process and outcomes. It is a reliable and an equal proof of value for everyone. Theoretically, blockchain can solve the problems of information asymmetry and trust among 
strangers because of its decentralization and immutability. It ensures the authenticity because the information and value are published and maintained collectively. It provides a trustworthy way for talent investment. The user with more education on digital currencies has much chance to win the appreciation and investment. Blockchain ledger tracks everything you've ever learned. Employers can use this information to offer you a job that matches your skills. On the other hand, the user who wants an excellent employee can also resort to the blockchain ledger. It will greatly decrease the risk of investment bias and failure. In a word, blockchain maximizes the interests of both parties.

\section{Potential issues of applying blockchain technology in education}

It is undeniable that there are potential drawbacks of applying blockchain technology in education.

As a complex system, some learning behaviors and learning outcomes need to be reviewed by the instructors subjectively such as essays and classroom presentations. It is quite hard to evaluate this kind of learning activities by the pre-programmed smart contract without human intervention.

If an educational blockchain system were put into use in schools, all students' educational data would be integrated into blockchain ledgers. The immutability feature of blockchain technology would act as a double-edged sword. It removes the possibility of modifying educational record for legitimate reasons for some students.

Furthermore, many technical issues or barriers are not addressed for the blockchain to be used in education. For example, the classic Proof of Work consensus mechanism wastes energy and has a poor performance in terms of number of transactions per second (Vukolić 2015), which would cost an extra expense, and hinder its application in schools.

\section{Conclusion}

Blockchain is essentially a distributed ledger technology, which uses the cryptograph techniques and distributed consensus algorithms to create the features of decentralization, traceability, immutability, and currency properties. It's currency properties has the potential to trigger many innovative applications for education. For example, by realizing "learning is earning," blockchain technology can foster students' learning motivation. It can store a complete, trustworthy set of record of educational activities including the processes and results in formal as well as informal learning environments. It can also record teachers' teaching behaviors and performance thus providing a reference for teaching evaluation. In a word, for both learners and teachers, blockchain has great potential applications in instructional design, behaviors recording, and analysis as well as formative evaluation. At the same time, it brings challenges and opportunities to researchers, developers, and educators.

For researchers, blockchain has excellent potential to be broadly applied in education. However, very few researches have been conducted. It would be challenging to study more closely on topics like, what opportunities can it offer for education revolution? How to better utilizing the digital currency property to enhance learning motivations and achievements? 
For developers, these creative ideas are just the first steps of applying blockchain into education. It is also an important part to develop educational platforms and software, which brings challenges for developers. How to build a blockchain platform that meets users' personalized needs? How to combine hardware with blockchain to create an environment for data acquisition and recording? How to maintain a massive volume of educational transaction data and integrate blockchain technology into the existing educational tools and systems?

For educators, some of the benefits of adopting blockchain technology to design smart contact-based learning activities are smart contract-based learning activities can be verifiable, robust, and traceable. This transparency feature is a strong protection for teachers who have done a good job. Furthermore, the school management regarding the evaluation of teaching performance should also be changed to embrace this new technology.

The realization of $21^{\text {st }}$ century's security, privacy, trust, and equality can be implemented by blockchain technology. Security refers to the protection of valuable properties and information. Nowadays some people have property but cannot prove ownership, such as intellectual property disputes. It may lead to conflict with others. Blockchain technology can be used by checking the records in the database to prove the properties. Some business information, such as design drawings, corporate planning, may be stolen by industrial spies. Blockchain technology can be used to protect these valuables business by recording data in a blockchain network. Blockchain technology protects teachers' instructional design from usurpation, thus improving the security of protecting intellectual properties.

Privacy means each node save the complete ledger, including all the information except for the real identity. For the sake of privacy, users' identifications are all presented by ID numbers. It means that blockchain technology protects the privacy of the trader, as no one else would have the private key. In the education scenario, all the information about learning experience recorded on blockchain can only be gained by its unique user's private key. Others are not accessible, which means blockchain users' privacy can be well guaranteed.

As for trust, blockchain technology can transform people's ways of constructing trust from building it by the third-party institution to building it by technology. Teachers' and students' behaviors are both recorded and monitored when smart contract and blockchain are applied. The trust between the subjects is based on the technology itself, not the third-party.

Equality refers to the equal rights and opportunities that everyone has on a blockchain network. The openness, borderless and permissionless natures of blockchain technology can provide everyone equal access to the technology and the network built with it. Anyone can apply for an electronic wallet on blockchain network. Blockchain technology does not set any limits for the users. All schools, teachers, and students can apply it to daily, thus avoiding authority-bias.

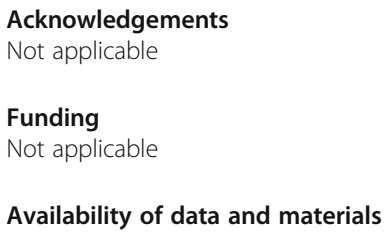




\section{Authors' information}

Dr. Guang Chen is Assistant Professor in the School of Educational Technology, Faculty of Education, Beijing Normal University. His current research interest is cognitive development in information age and smart learning environments. Bing Xu is a Master in the School of Educational Technology, Faculty of Education, Beijing Normal University. Her current research interest is smart learning environments and digital reading.

Manli Lu is a Master in the School of Educational Technology, Faculty of Education, Beijing Normal University. Her current research interest is online synchronous learning and smart learning environments.

Dr. Nian-Shing Chen is Chair Professor in the Department of Information Management at the National Sun Yat-sen University, Taiwan. His current research interests include assessing e-Learning course performance; online synchronous teaching \& learning; mobile \& ubiquitous learning; Natural user interface \& Game-based learning.

\section{Consent for publication}

Neither this manuscript nor any significant part of it is under consideration for publication elsewhere or published or available elsewhere in a manner that could be construed as a prior or duplicate publication of the same or substantially overlapping content.

\section{Competing interests}

The authors declare that they have no competing interests.

\section{Publisher's Note}

Springer Nature remains neutral with regard to jurisdictional claims in published maps and institutional affiliations.

\section{Author details}

${ }^{1}$ School of Educational Technology, Faculty of Education, Beijing Normal University, Beijing, China. ${ }^{2}$ Beijing Key Laboratory of Education Technology, Beijing Normal University, Beijing, China. ${ }^{3}$ Department of Information Management, National Sun Yat-sen University, Kaohsiung, Taiwan.

Received: 5 September 2017 Accepted: 21 December 2017

Published online: 03 January 2018

\section{References}

R Beck, JS Czepluch, N Lollike, S Malone, Blockchain - The Gateway to Trust-Free Cryptographic Transactions. In Research Papers from ECIS2016, (Istanbul, 2016)

M Chung, J Kim, The internet information and technology research directions based on the fourth industrial revolution. KSII Trans. Internet Inf. Syst. 10(3), 1311-1320 (2016)

R Collins, Blockchain: A new architecture for digital content. EContent 39(8), $22-23$ (2016)

$P$ Devine, Blockchain learning: can crypto-currency methods be appropriated to enhance online learning? Presented at the ALT Online Winter Conference 2015, Online, (United Kingdom, 2015)

K Fanning, DP Centers, Blockchain and its coming impact on financial services. J. Corp. Account. Finance 27(5), 53-57 (2016) https://doi.org/10.1002/jcaf.22179

S Haber, WS Stornetta, How to time-stamp a digital document. J. Cryptol. 3(2), 99-111 (1991) https://doi.org/10. 1007/BF00196791

MB Hoy, An introduction to the Blockchain and its implications for libraries and medicine. Med. Ref. Serv. Q. 36(3), 273-279 (2017) https:/doi.org/10.1080/02763869.2017.1332261

A Kosba, A Miller, E Shi, Z Wen, C Papamanthou, in 2016 IEEE Symposium on Security and Privacy (SP). Hawk: The Blockchain Model of Cryptography and Privacy-Preserving Smart Contracts (2016), pp. 839-858 https://doi.org/10.1109/SP.2016.55

D Kraft, Difficulty control for blockchain-based consensus systems. Peer Peer Netw. Appl. 9(2), 397-413 (2016) https://doi.org/10.1007/s12083-015-0347-x

S Nakamoto, Bitcoin: A peer-to-peer electronic cash system. (2008) Retrieved from https://bitcoin.org/bitcoin.pdf

M Peck, A blockchain currency that beat s bitcoin on privacy. IEEE Spectr. 53(12), 11-13 (2016) https://doi.org/10.1109/MSPEC.2016.7761864

K Schwab, The Fourth Industrial Revolution (The Crown Publishing Group, New York City, NY, 2017)

M Sharples, J Domingue, in The Blockchain and Kudos: A Distributed System for Educational Record, Reputation and Reward. Adaptive and adaptable learning (Springer, Cham, 2016), pp. 490-496 https:/doi.org/10.1007/978-3-319-45153-4_48

DJ Skiba, The potential of Blockchain in education and health care. Nurs. Educ. Perspect. 38(4), 220-221 (2017) https://doi.org/10.1097/01.NEP.0000000000000190

M Swan, Blockchain: Blueprint for a New Economy, 1st edn. (O'Reilly Media, Sebastopol, CA, 2015)

F Tschorsch, B Scheuermann, Bitcoin and beyond: A technical survey on decentralized digital currencies. IEEE Commun. Surv. Tutorials 18(3), 2084-2123 (2016) https://doi.org/10.1109/COMST.2016.2535718

S Underwood, Blockchain beyond Bitcoin. Commun. ACM 59(11), 15-17 (2016) https://doi.org/10.1145/2994581

M Vukolić, in The Quest for Scalable Blockchain Fabric: Proof-of-Work vs. BFT Replication. Open problems in network security (Springer, Cham, 2015), pp. 112-125 https://doi.org/10.1007/978-3-319-39028-4_9

H Wang, K Chen, D Xu, A maturity model for blockchain adoption. Financ. Innov. 2(1), 12 (2016) https://doi.org/10.1186/s40854-016-0031-z

J Yli-Huumo, D Ko, S Choi, S Park, K Smolander, Where is current research on Blockchain technology?-A systematic review. PLoS One 11(10), e0163477 (2016) https://doi.org/10.1371/journal.pone.0163477

Z Zheng, S Xie, H Dai, X Chen, H Wang, in 2017 IEEE International Congress on Big Data (BigData Congress). An Overview of Blockchain Technology: Architecture, Consensus, and Future Trends (2017), pp. 557-564 https://doi.org/10.1109/ BigDataCongress.2017.85 